\title{
Comunidades de arañas en dos localidades del sitio RAMSAR Humedales Chaco, Argentina
}

\author{
Helga Cecilia Achitte Schmutzler, Gilberto Avalos \& Elena Beatriz Oscherov \\ Cátedra de Biología de los Artrópodos, Facultad de Ciencias Exactas y Naturales, Universidad Nacional del Nordeste, Avda. Libertad 5470 \\ (3400) Corrientes, Argentina; gilbertoa@exa.unne.edu.ar; ceciliaachitte@hotmail.com; eboscherov@yahoo.com.ar
}

Recibido 12-III-2016 • Corregido 17-V-2016 • Aceptado 10-VII-2016

\begin{abstract}
Spider communities in two localities of the RAMSAR Site Chaco Wetlands, Argentina. The RAMSAR Site, Chaco wetlands, covers the eastern fringe of the Chaco province, with a surface of 508000 ha and several environmental unit, like gallery forest, floodplain forests, palm savannahs, grasslands, and others. The studies about the spider fauna from its site are scarce. This study compares spider communities from forest and grassland in San Francisco ranch (27030'26" S - 59 $05^{\prime} 03^{\prime \prime}$ W) located in San Fernando Department and San Carlos ranch (2657"46" S - 58038'12" W) located in Bermejo Department; on October and November 2013. The forest structure is different, San Francisco forest is mixed with Chaco vegetation and the cattle activity has destroyed an important part of it. The San Carlos forest is a forest gallery also constituted by paranaense species, with cattle activity too, but it with more control and carefulness by their owners. The spiders were collected by foliage beating, leaf litter sifting, hand collecting and G-vac (vacuum sampling) sampling methods. A total of 1477 spiders grouped into 261 species/morphospecies from 34 families of Araneomorphae. Eighteen species are new records for Argentina. Araneidae was the most abundant families. Araneidae, Theridiidae and Salticidae were the most richness families. Species accumulation curves with 95\% confidence interval show that there was significant difference only for grasslands. Rank abundance curve showed that community structure were different on both sites, referring to abundance, dominant species, species composition and proportion of rare species. The comparison of true diversity showed that San Carlos grassland and forest were more diverse than San Francisco, ones it could be related to vegetation structure and high degree of conservation. This study showed an important richness and abundance of spiders that integrate different communities on RAMSAR site areas, and suggests future research on others environments on this site.
\end{abstract}

Key words: Araneae, diversity, forest, grassland, priority area, Chaco Wetlands, Argentina.
RESUMEN: En este estudio comparamos las comunidades de arañas de bosques y pastizales en la Estancia San Francisco y la Estancia San Carlos; Sitio RAMSAR, Humedales Chaco. Recolectamos los ejemplares de arañas mediante las técnicas de captura golpeteo de follaje, tamizado de hojarasca, captura directa y aspirado del pastizal. Se obtuvieron 1477 arañas, 34 familias y 261 especies/morfoespecies de Araneomorphae. 18 son nuevos registros para Argentina. Araneidae fue la más abundante en los dos sitios, Araneidae, Theridiidae y Salticidae las de mayor riqueza específica. Las curvas de rango-abundancia marcaron que las comunidades se estructuran de manera diferente en ambos sitios, en cuanto a las abundancias, el tipo de especie dominante, la composición de especies y la proporción de especies raras. La comparación de la diversidad verdadera mostró que el bosque y pastizal de San Carlos fueron más diversos, lo cual podría estar relacionada a la estructura del bosque y a un mayor grado de conservación del mismo. Este estudio mostró una importante riqueza y abundancia de arañas que componen comunidades diferentes en estas áreas del sitio RAMSAR, y sugiere profundizar en investigaciones futuras abarcando otras unidades ambientales de dicho sitio.

Palabras clave: Araneae, diversidad, bosque, pastizal, área prioritaria, Humedales Chaco, Argentina.
En los ecosistemas terrestre las arañas desarrollan roles importantes. Debido a su gran abundancia y sus hábitos alimenticios, influyen en el equilibrio ecológico (Gertsch, 1979; Young \& Edwards, 1990; Wise, 1993). Se alimentan principalmente de insectos y de otras arañas
(Nyffeler, 1999). Como se encuentran entre los más abundantes de la fauna macropredadora terrestre (Swift et al., 1979; Wise, 1993), estabilizan y regulan las poblaciones de artrópodos de diferentes niveles tróficos (Symondson et al., 2002; Wise et al., 2006). Además, algunas especies 
se alimentan de huevos, larvas e insectos considerados plagas para los cultivos (Young \& Edwards, 1990) lo cual otorga gran importancia a las arañas como reguladores biológicos (Green, 1996).

La complejidad estructural de un hábitat incide en la composición de familia de arañas, dado que las familias del mismo gremio buscan estar en ambientes similares (Whitmore et al., 2002). Asimismo, alteraciones en el hábitat pueden limitar las poblaciones de las especies cuya estrategia de caza depende de construcciones de telas al disminuir los sitios para fijarlas (Colebourne, 1974; Riechert \& Cady, 1983; Riechert \& Gillespie, 1986). También juega un rol importante la demanda espacial, así, la dispersión y forma de crecimiento de los sustratos vegetales podrían ser pocos adecuados para el anclaje de la telas, hechos que pueden incidir en la baja riqueza y abundancia de arañas orbitelares en el bosque (Janetos, 1986; Marshall \& Rypstra, 1999)

Los Humedales del Chaco argentino, declarados Sitio RAMSAR, abarca 508 mil ha en la zona Oriental del Chaco y se encuentra en la Región biogeográfica del Chaco Húmedo. Dicho Sitio, presenta una gran variedad de unidades ambientales, bosques en galería, bosques bajo inundable, sabana palmar, pastizales, zonas anegables dedicadas a la ganadería y zonas dedicadas a la agricultura. Se estima que alberga distintas composiciones de la araneofauna, que hasta el momento ha sido poco estudiada.

Estudios realizados en la Región Chaco Húmedo sobre comunidades de bosques y pastizales, dan cuenta de la gran abundancia de familias de Araneae, entre ellos se destacan los estudios de ambientes naturales efectuados en el Chaco (Bar et al., 2008) y en Corrientes (Avalos et al., 2007), como así también las realizadas en áreas naturales protegidas, como en la Reserva Colonia Benítez, Chaco (Escobar et al., 2012), de la provincia de Formosa (Corronca \& Abdala, 1994), y en Corrientes (Avalos et al., 2009; Rubio et al., 2008).

Este trabajo tuvo como objetivo caracterizar la fauna de arañas del Sitio RAMSAR, analizar comparativamente la diversidad, riqueza específica y abundancia, tanto de bosques como de pastizales en dos áreas.

\section{MÉTODOS}

Área de estudio: Los muestreos los realizamos en octubre y noviembre de 2013, en la Estancia San Francisco, (27030'26" S-59 05'03" W) ubicada en el Departamento San Fernando, y la Estancia San Carlos (265" $46^{\prime \prime} \mathrm{S}-58^{\circ} 38^{\prime} 12^{\prime \prime} \mathrm{W}$ ) en el Departamento Bermejo.
El área de estudio integra la lista RAMSAR con el nombre de Humedales Chaco, Argentina, desde febrero de 2004. El sitio RAMSAR, de $153 \mathrm{Km}$ de longitud, abarca la franja oriental de la Provincia del Chaco, e incluye los Departamentos San Fernando, Primero de Mayo y Bermejo. Limita al Norte por el cauce del Río Bermejo, al Sur el paralelo $28^{\circ}$, al Este por los cauces de los ríos Paraná y Paraguay y al Oeste por el trazado de la Ruta Nacional NN11. Biogeográficamente el área está incluida en la Región Neotropical, Subregión Chaqueña, que corresponde el centro y norte de Argentina, Sur de Bolivia, oeste de Paraguay y sur este de Brasil (Morrone, 2001).

Actividades de campo: En cada ambiente (bosque y pastizal) de la Estancia San Francisco (S.F.) y San Carlos (S.C.), trazamos tres transectos de $200 \mathrm{~m}$ de longitud, en cada uno seleccionamos cinco puntos separados entre sí por 50m., donde se tomaron muestras aplicando las siguientes técnicas de captura:

a. Golpeteo de follaje: consistió en 15 golpes sobre la vegetación arbustiva y en la porción baja del estrato arbóreo, el material recolectamos sobre un lienzo blanco de $2,50 \mathrm{~m}^{2}$.

b. Tamizado: tamizamos una superficie de $0,50 \mathrm{~m}^{2}$ de hojarasca, con un tamiz de $10 \times 15 \mathrm{~mm}$ de apertura de malla.

c. Captura directa: efectuamos capturas manuales nocturnas, que se llevaron a cabo por dos personas durante una hora. Tanto en el bosque como en el pastizal.

d. Aspirado: lo realizamos mediante el uso de una aspiradora de jardín (Mod. 220 V-AR).

Totalizamos 45 muestras en el bosque y 30 en el pastizal; todas las muestras las consideramos independientes y el material obtenido lo conservamos en alcohol etílico $70 \%$ para su transporte al laboratorio.

Actividades de laboratorio: Los ejemplares capturados los identificamos mediante claves específicas, a nivel de familia, género y especie en los casos posibles. Los individuos que no pudimos determinar a nivel específico, los clasificamos como morfoespecies según sus características estructurales fenotípicas. Dicha determinación es respaldada por la parataxonomía para una identificación más rápida (Krell, 2004).

Las arañas fueron separadas por estado del desarrollo, sexo y clasificadas por gremios (Enders, 1976; DippenaarSchoeman et al., 1989; Uetz et al., 1999) en constructoras 
de telas orbiculares $=$ CTO, constructoras de telas sábanas $=C T S$, vagabundas de vegetación $=V \mathrm{~V}$, vagabundas del suelo $=$ VS, cazadoras al acecho $=C A$, cazadoras por emboscada $=C E$, constructoras de telas espaciales $=C T E$ y constructoras de telas tubulares $=$ CTT.

Todos los ejemplares los depositamos en la colección CARTROUNNE del Laboratorio de Artrópodos, Facultad de Ciencias Exactas y Naturales y Agrimensura, UNNE.

Análisis de datos: Para determinar la representatividad del muestreo, elaboramos curvas de acumulación de especies para cada ambiente (Moreno, 2001), para lo cual se utilizaron los estimadores no paramétricos Ace y Chaol que se basan en la abundancia (JiménezValverde \& Hortal, 2003), Jacknife 1 que no asume la homogeneidad ambiental en la muestra, y Bootstrap que estima la riqueza en ensambles con gran cantidad de especies raras (Magurran, 2004). La curva de acumulación de especies fue suavizada mediante el reordenamiento aleatorio repetido de las muestras (100 repeticiones) (Longino \& Colwell, 1997).

La diversidad de ambas comunidades las medimos mediante el número efectivo de especies, entendido como el número de especies de una comunidad virtual, perfectamente balanceada, en la que todas las especies son igualmente comunes, y en la cual se conserva la abundancia relativa promedio de las especies de la comunidad real (Jost, 2006). Los números efectivos de especies permiten comparar de forma clara y directa la magnitud de la diferenciación en la diversidad de dos o más comunidades (Jost, 2006; Moreno et al., 2011).

En este trabajo consideramos las medidas de diversidad verdadera de orden cero $(0 \mathrm{D}=\mathrm{S})$, cuyo valor equivale a la riqueza de especies, y de orden uno (1D), en la cual todas las especies son consideradas en el valor de diversidad, ponderadas proporcionalmente según su abundancia en la comunidad (Hill, 1973; Jost, 2006, 2007; Moreno et al., 2011). Además, la diferencia en la diversidad verdadera entre comunidades se expresó como el cociente entre la de mayor diversidad respecto a la de menor diversidad, a fin de determinar cuánto más diversa es una comunidad comparada con otra.
Los distintos ambientes se compararon mediante las curvas de rango-abundancia, cuya gráfica permite analizar la riqueza de especies, sus abundancias relativas, la equitatividad y la secuencia de cada una de las especies que componen la comunidad (Feinsinger, 2004). Se utilizó para los análisis estadísticos el programa Estimates 9.1 (Colwell, 2013).

\section{RESULTADOS}

Obtuvimos en total 1477 arañas, pertenecientes a 34 familias de Araneomorphae y 261 especies/morfoespecies. En S.F. se registraron 30 familias y 147 especies, y en S.C. 26 familias y 167 especies. Amphinectidae, Deinopidae, Hahniidae y Uloboridae solo se registraron en S.C. y, en S.F., Dictynidae, Eutichuridae, Miturgidae, Pisauridae, Scytodidae, Titanoecidae, Pholcidae y Trechaleidae.

La familia con mayor número de individuos fue Araneidae en las dos áreas de estudio. Las familias Araneidae y Theridiidae presentaron la mayor riqueza en el bosque de S.F. con igual número de especie y Salticidae para el pastizal. En S. C. Araneidae presentó la mayor riqueza específica tanto en el bosque como en el pastizal (Apéndice digital 1).

Las curvas de acumulación de especies en los ambientes muestreados no llegaron a la asíntota (Fig. 1), sin embargo de acuerdo a los estimadores calculados, el esfuerzo de muestreo fue suficiente en bosques de las dos áreas de estudios y en pastizal de S.C., ya que las especies esperadas están representadas entre un 50 y $80 \%$. Por otra parte, en el pastizal de S.F. según los modelos Ace y Chao1, los muestreos fueron insuficientes (Cuadro 1).

Los valores de riqueza específica no fueron significativamente diferentes entre ambos bosques, dado que los intervalos de confianza del $95 \%$, para la curva de acumulación de especies, se superponen. No obstante en los pastizales no hubo una superposición de los intervalos, lo que implica que son significativamente diferentes (apéndice digital 2).

Los resultados de la Diversidad verdadera, según los números efectivos de especies (Cuadro 2), mostraron

CUADRO 1

Riqueza de especies observadas y esperadas en cada ambiente según los estimadores calculados

\begin{tabular}{|c|c|c|c|c|c|c|}
\hline & & Sobs & ACE (\%) & CHAO1 (\%) & JACK1 (\%) & Bootstrap (\%) \\
\hline San & Bosque & 103 & 66 & 65 & 68 & 83 \\
\hline Francisco & Pastizal & 43 & 23 & 27 & 58 & 78 \\
\hline San & Bosque & 111 & 74 & 75 & 71 & 84 \\
\hline Carlos & Pastizal & 69 & 57 & 53 & 65 & 82 \\
\hline
\end{tabular}



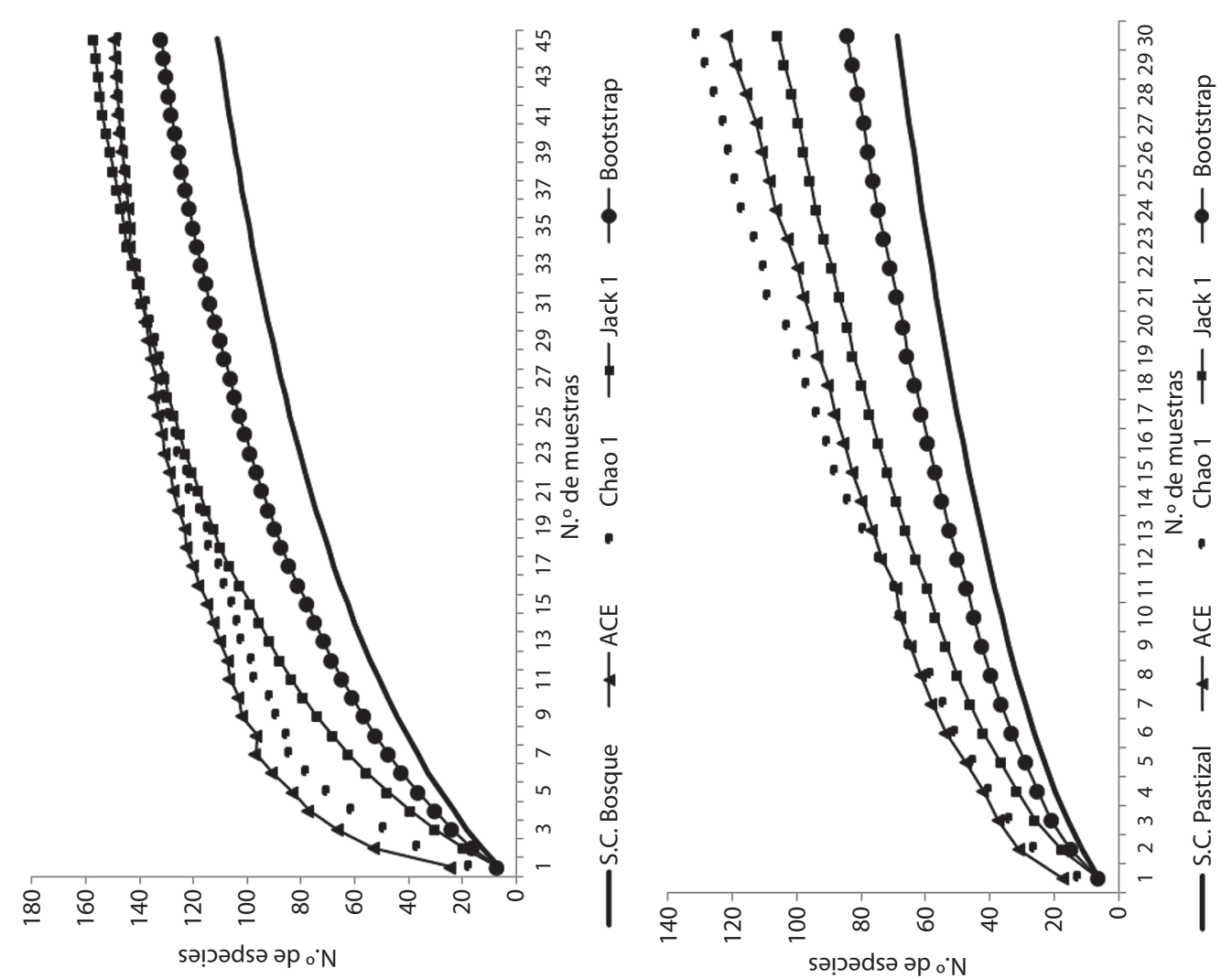

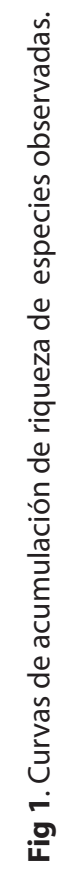
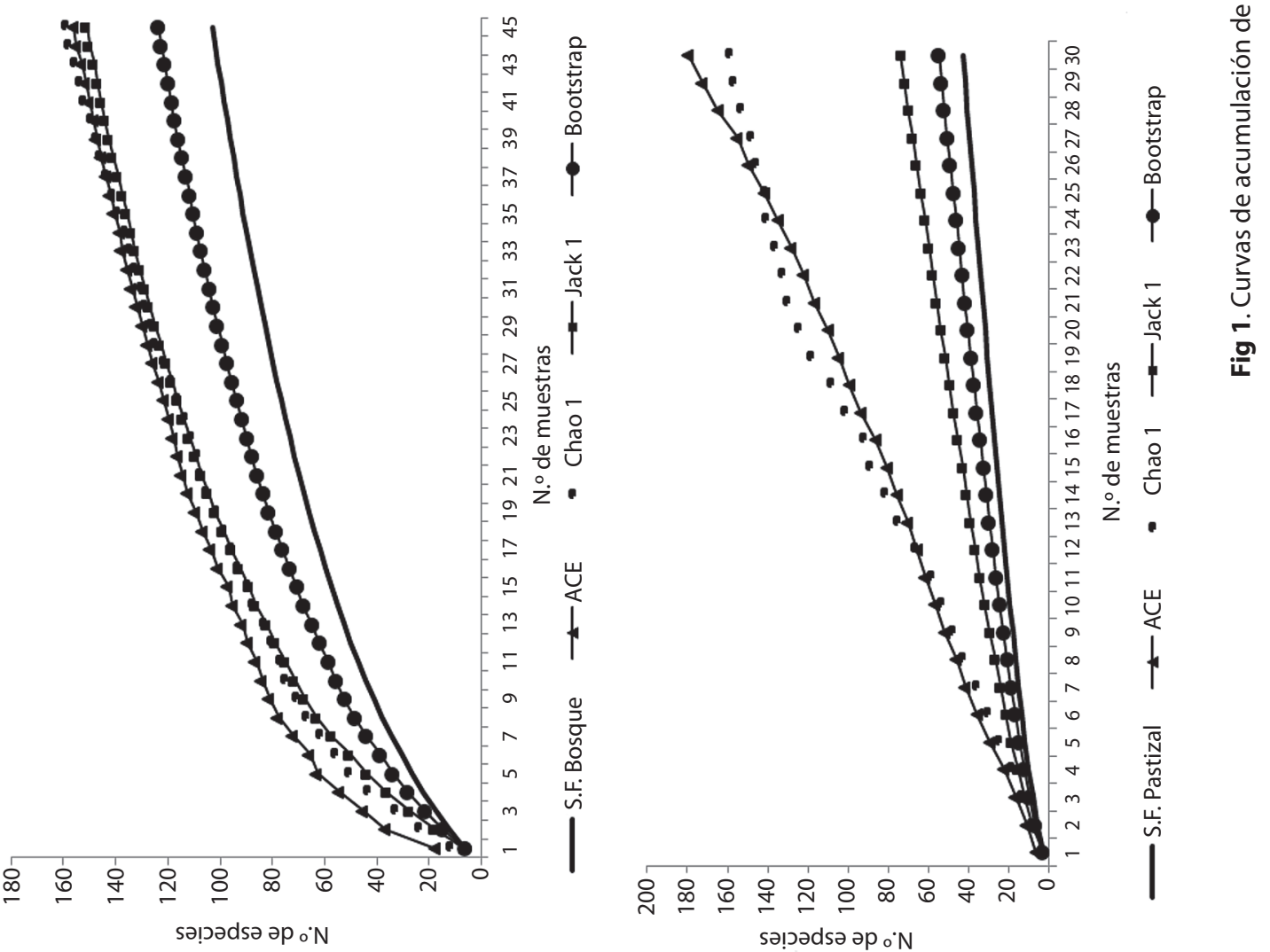
que el bosque de S.C. es 1,35 veces más diverso que el de S.F. En el mismo sentido se verificó que el pastizal es 2,43 más diverso en el primero que el segundo.

CUADRO 2

Medidas de diversidad verdadera de orden cero $\left({ }^{0} \mathrm{D}\right)$ y de orden uno ( $\left.{ }^{1} D\right)$

\begin{tabular}{lcccc} 
& \multicolumn{2}{c}{ San Francisco } & \multicolumn{2}{c}{ San Carlos } \\
Ambientes & ${ }^{0} \mathrm{D}$ & ${ }^{1} \mathrm{D}$ & ${ }^{0} \mathrm{D}$ & ${ }^{1} \mathrm{D}$ \\
Bosque & 103 & 56,26 & 111 & 75,19 \\
Pastizal & 43 & 10,59 & 69 & 25,79 \\
\hline
\end{tabular}

Las curvas de abundancia relativa del total de las especies (apéndice digital 3) mostraron un gran número de especies raras en los sitios de muestreo. Así en S.F. se verificaron 27 especies con un individuo (singletons) y 36 especies con dos ejemplares (doubletons) observadas en 27 familias, mientras que en S.C. fueron 23 las familias con singletons (62) y doubletons (30).

Respecto al patrón de distribución y el orden jerárquico de las especies, los bosques presentaron una pendiente poco pronunciada, con una especie dominante, Micrathena swainsoni (Araneidae) en S.F. y Parawixia audax (Araneidae) en S.C. En los pastizales, las pendientes presentaron una mayor inclinación lo que significa una menor equitatividad en las comunidades. Particularmente, el pastizal de S.C. mostró menor grado de variación entre las abundancias de las especies que el de S.F., por tanto fue el más equitativo. Alpaida venilae (Araneidae) fue la especie dominante para estos ambientes. (apéndice digital 3)

Teniendo en cuenta las diez especies más abundantes (apéndice digital 4), en bosques de S.F., las especies pertenecen al gremio CTO: Micrathena swainsoni, Alpaida bicornuta, Micrathena sp1 y Parawixia sp1 (Araneidae), CTS: Sphecozone gravis (Linyphiidae), CA: Semiopyla viperina, morfoespecie 4 (Salticidae), CE: Tmarus estyliferus (Thomisidae), VS: Trachelopachys aemulatus (Trachelidae) y VV: Osoriella tahela (Anyphaenidae). En S.C., las especies son del gremio CTO: Parawixia audax, Acacesia tenella, Eustala sp5, Mangora sp2 (Araneidae), Leucauge sp1 (Tetragnathidae), Miagrammopes sp (Uloboridae), CTS: Dubiaranea sp (Linyphidae) y CTE: Chrysso sp, Morfoespecie 7 (Theridiidae) y VV: Osoriella tahela (Anyphaenidae).

Respecto a los pastizales, también las especies que se destacan son las del gremio CTO: Alpaida venilae, Ocrepeira hirsuta y Eustala sp2 (Araneidae), CTS: Tutaibo velox (Linyphiidae), CA: Aphirape gamas, Morfoespecie 9 (Salticidae), Oxyopes salticus (Oxyopidae); CE: Tibellus paraguensis, Tibellus sp (Philodromidae); VV: Aysha caxambuensis (Anyphaenidae) en S.F.; y CTO: Alpaida venilae, Ocrepeira hirsuta y Neoscona moreli, CA: Aphirape gamas; CE: Tibellus paraguensis; VV: Arachosia proseni, Osoriella tahela, Arachosia sp (Anyphaenidae) y VS: Morfoespecie 1 y 2 (Lycosidae) en S.C.

En este trabajo se citamos 18 especies nuevas para Argentina, las cuales se nombran a continuación: Castianeira buelowae, Camillina mahnerti, Eilica bicolor, Sphecozone gravis, Tutaibo velox, Lycosa carboneIli, Gelanor insularis, Oxyopes salticus, Cotinusa vittata, Faiditus alticeps, Janula erythrophthalma, Cryptachaea rioensis, Thwaitesia bracteata, Guaraniella mahnerti, Tmarus estyliferus, Tobias paraguayensis, Trachelopachy gracilis, Trachelopachy aemulatus.

\section{DISCUSIÓN}

El número de familias halladas en esta investigación representa el $49 \%$ de las registradas en Argentina, y en referencia al número de especies que se conocen para este País, representan el $21 \%$ de las citadas actualmente (Grismado \& Goloboff, 2014) .

El total de las familias recolectadas (34), es importante para el Chaco Húmedo si se tiene en cuenta las obtenidas por Escobar et al. (2012) en la Reserva Provincial de Colonia Benítez, Chaco (27 familias), en la Reserva Provincial del Iberá (33 familias), Avalos et al. (2009) y en dos bosques en el Departamento Capital, Corrientes: El Perichon (30 familias) y Laguna Brava (26 familias).

En coincidencia con Escobar et al. (2012) y Avalos et al. (2007) Araneidae fue la familia con mayor número de individuos en ambas áreas. Esta abundancia marcada de este grupo de araña, probablemente esté relacionada con la complejidad estructural de los bosques y los altos pastizales que se encuentra en el Sitio RAMSAR. Concretamente, en el bosque de S.C., esta familia además fue la de mayor riqueza específica, lo que presume su mayor estado de conservación, siguiendo a Riechert y Gillespie (1986) quienes relacionan la alta diversidad y abundancia de arañas constructoras de telas orbiculares con la cantidad de estructuras disponibles y dispersión de las mismas en el hábitat, utilizadas para la fijación de las telas.

La elevada riqueza de Araneidae, Theridiidae y Salticidae encontrada en este estudio, es de esperarse al tratarse de familias con alto número de especies a nivel mundial, tan solo en nuestro País, Grismado y Goloboff (2014) mencionan 188 especies para Salticidae, 173 para Araneidae y 91 para Theridiidae. 
Respecto a los altos valores de singletons y doubletons, registradas en los sitios de muestreos, coincide con lo señalado por New (1999), que el número de especies raras en los inventarios de arañas son elevadas. Además, Jiménez-Valverde y Hortal (2003) aseguran que aunque el estudio se limite a una sola familia de Araneae, la complejidad de su composición conlleva al gran número de especies raras y a un inventario incompleto.

En general las curvas de acumulación de especies registradas en este trabajo denotan una colecta entre el $60 \%$ y $80 \%$ en los ambientes analizados, sin llegar a la asíntota. Jiménez-Valderde y Hortal (2003) aseveran que generalmente ningún trabajo sobre la araneofauna llegan a registrar la totalidad de especies, por lo que la curva no llega a la asíntota, por ende no existen criterios objetivos que permitan decir cuando un muestreo es suficientemente completo. Además, Longino et al. (2002) alegan incertidumbre a la veracidad de los estimadores de riqueza utilizados para evaluar la completitud de los inventarios, ya que se encuentran en un período de experimentación.

Si bien las curvas de acumulación de especies, con intervalos de confianza del 95\%, mostraron que ambos bosques muestreados son similares; en San Carlos, a pesar del menor número de familias encontradas, fue donde se obtuvo la mayor riqueza de especies, asimismo estos valores concuerdan con los resultados arrojados con el cálculo del número efectivo de especies. Además las curvas de rango-abundancia mostraron diferencias entre los ambientes. El análisis de las abundancias, el tipo de especie dominante, la composición de especies y la proporción de especies raras, demuestran que las comunidades se estructuran de forma diferente. La elevada diversidad registrada en San Carlos, puede estar relacionada con la estructura propia del lugar, que corresponde a una selva en galería, según Chebez (2009), las selvas del este de Formosa y Chaco presentan valores elevados de diversidad solo superado por las selvas misioneras en nuestro país. A diferencia de San Francisco cuyo ambiente corresponde a un bosque degradado por la presencia de ganado y cuya conformación vegetal es diferente. Este aspecto es valioso considerar porque la conformación física del ambiente limita la composición de las comunidades de arañas, al influir en los sitios de refugio, reproducción y alimentación. Este estudio mostró una importante riqueza y abundancia de arañas en estas áreas del sitio RAMSAR, y sugiere profundizar en investigaciones futuras abarcando otras unidades ambientales de dicho sitio.

\section{AGRADECIMIENTOS}

Este trabajo fue financiado por la ANPCyT (PICTOUNNE 2011-0244) y la Secretaría de Ciencia y Técnica de la Universidad Nacional del Nordeste (PI F005/11).

\section{REFERENCIAS}

Avalos, G., Rubio, G. D., Bar, M.E., \& González, A. (2007). Arañas (Arachnida, Araneae) asociadas a dos bosques degradados del Chaco húmedo en Corrientes, Argentina. Revista de Biología. Tropical, 55, 899-909.

Avalos, G., Damborsky, M. P., Bar, M. E., Oscherov, E. B., \& Porcel, E. (2009). Composición de la fauna de Araneae (Arachnida) de la Reserva Provincial Iberá, Corrientes, Argentina. Revista de Biología Tropical, 57 (1-2), 339-351.

Bar, M. E., Oscherov, E.B, Damborsky, M. P., Avalos, G., \& Núñez, E. (2008). Primer inventario de la fauna de Arthropoda de la Región Chaqueña Húmeda. INSUGEO Miscelánea, $17,331-353$.

Chebez, J.C. (2009) Los que se van. Ecorregiones de la Argentina II: las selvas en galería. Recuperado de: http://www.losquesevan.com/ecorregiones-de-la-argentina-ii-las-selvas-en-galeria.74c.

Colebourne, P. H., (1974). The influence of habitat structure on the distribution of Araneus diadematus. Journal of Animal Ecology, 43, 401-409.

Colwell, R. K., (2013). EstimateS: Statistical estimation of species richness and shared species from samples. (Version 9.1.0). User's guide and application: http://viceroy.eeb. uconn.edu/estimates.

Corronca, J.A., \& Abdala, C.S. (1994). La fauna araneológica de la Reserva Ecológica "El Bagual", Formosa, Argentina. Nota preliminar. Aracnología, 9, 1-6.

Dippenaar-Schoeman, A.S., van den Berg, A.M, \& van den Berg, A., (1989). Species composition and relative seasonal abundance of spiders from the field and tree layers of the Roodeplaat Dam Nature Reserve. Koedoe 32, 25-38.

Enders, F. (1976). Clutch size related to hunting manner of Spider species. Annals of the Entomological Society of America, 69, 991- 998.

Escobar, M. J.; Avalos, G., \& Damborsky, M. P. (2012). Diversidad de Araneae (Arachnida) en la Reserva Colonia Benítez, Chaco Oriental Húmedo, Argentina. Revista FACENA, 28, 3-17.

Feinsinger, P. (2004). El diseño de estudios de campo para la conservación de la biodiversidad. Editorial FAN, Santa Cruz. 242 p.

Gertsch, W. J. (1979). American spiders (2nd ed.). New York, Van Nostrand.

Grismado, C., \&. Goloboff, P. A. (2014). Rastelloidina. En: Roig-Juñent, S.; L.E. Claps \& J.J. Morrone (Directores), Biodiversidad de Artrópodos Argentinos volumen 3, 
pp. 103-110, Editorial INSUE - UNT, San Miguel de Tucumán, Argentina.

Green, J. (1996). Spiders in biological control- An Australian perspective. Revue Suisse de Zoologie Volume. hors série: $245-253$.

Hill, M. O. (1973). Diversity and evenness: a unifying notation and its consequences. Ecology, 54,427-432.

Janetos, A., (1986). Web- site selection: are we asking the right questions?: 9-22 (en) SHEAR, W., Spiders webs, behavior, and evolution. Stanford University Press, California

Jiménez-Valverde, A. \& Hortal J. (2003). La curva de acumulación de especies y la necesidad de evaluar los inventarios biológicos. Revista Ibérica de Aracnología, 8,151-161.

Jost, L. (2006). Entropy and diversity. Oikos, 113, 363-375.

Jost, L. (2007). Partitioning diversity into independent alpha and beta components. Ecology, 88, 2427-2439.

Krell, F.T. (2004). Parataxonomy versus taxonomy in biodiversity studies - pitfalls and applicability of 'morphospecies' sorting. Entomology, Strength in Diversity, XXII International Congress of Entomology, 15-21 August 2004, Brisbane Queensland Australia.

Longino, J. T., \& Colwell, R. K. (1997). Biodiversity assessment using structures inventory: capturing the ant fauna of a tropical rain forest. Ecological Applications, 7(4), 1263-1277.

Longino, J. T., Coddington J., \& Colwell, R. K. (2002). The ant fauna of a tropical rainforest: estimating species richness in three different ways. Ecology 83, 689-702.

Magurran, A. E. (2004). Measuring Biological Diversity. Oxford: Blackwell Publishing.

Marshall, S., \& Rypstra, A. (1999). Spider competition in structurally simple ecosystems. The journal of Arachnology, 27, 343- 350 .

Moreno, C. E. (2001). Métodos para medir la biodiversidad. M \& T-Manuales \& Tesis. SEA, Zaragoza, España. 83 p.

Moreno, C. E., Barragán, F., Pineda, E., \& Pavón, N. P. (2011). Reanálisis de la diversidad alfa: alternativas para interpretar y comparar información sobre comunidades ecológicas. Revista Mexicana de Biodiversidad, 82, 1249-1261.
Morrone, J. J. (2001) Biogeografía de América Latina y el Caribe. Manuales y Tesis. Sociedad Entomológica Aragonesa (SEA), 3, Zaragoza, España, 148 pp.

New, T.R. (1999). Untangling the web: spiders and the challenges of invertebrate conservation. Journal of Insect Conservation, 3, 251-256.

Nyffeler, M. (1999). Prey selection of spiders in the field. The Journal of Arachnology, 27, 317-324.

Riechert, S., \& Cady, A., (1983). Patterns of resource use and tests for competitive release in a spider community. Ecology, 64 (4), 899-913.

Riechert, S., \& Gillespie, R., (1986). Habitat choice and utilization in web-building spiders: 23-48 (en) SHEAR, W. Spiders webs, behavior, and evolution. Stanford University press, CaliforniaColebourne, 1974.

Rubio, G. D., Corronca, J., \& Damborsky M. P. (2008). Do spider diversity and assemblages change in different contiguous habitats? A case study in the protected habitats of the Humid Chaco ecoregión, north-east Argentina. Environmenntal Entomology. 37, 419-430.

Swift, M. J., Heal, O. W., Anderson, J. M., (1979). Decomposition in Terrestrial Ecosystems. University of California Press, Berkeley, CA, USA.

Symondson, W. O. C., Sunderland, K. D., \& Greenstone, M. H. (2002). Can generalist predators be effective biocontrol agents? Annual Review Entomology, 47, 561-594.

Uetz, G. W.,Halaj J,.\&. Cady, A. B. (1999). Pitfal traping in ecological studies of wandering spider. The Journal Arachnology, $27,270-280$.

Whitmore, C., Slotow, R., Crouch, T. E., \& Dippenaar-Schoeman, A. S. (2002). Diversity of spiders (Aranae) in a savanna, Northern province, South Africa. The Journal Arachnology, 30, 344-356.

Wise, D. H. (1993). Spider in Ecological Webs. Cambridge, Cambridge, Inglaterra. 328 p.

Wise, D. H., Modenhauer, D. M., \& Halaj, J. (2006): Using stable isotopes to reveal shifts in prey consumption by generalist predators. Ecological Applications, 16, 865-876.

Young, O. P., \& Edwards, G. B. (1990). Spiders in United States field crops and their potential effect on crop pests. The Journal Arachnology, 18, 1-27.

See Digital Appendix / Ver Apéndice digital 
